Journal of Applied Crystallography

ISSN 0021-8898

\section{A laboratory transmission diffraction Laue setup to evaluate single crystal quality}

\author{
Alexiane Arnaud, ${ }^{\mathrm{a}, \mathrm{b}}$ Wijdène Guediche, ${ }^{\mathrm{b}}$ Clément Remacha, ${ }^{\mathrm{a}}$ Edward Romero ${ }^{\mathrm{a}}$ \\ and Henry Proudhon ${ }^{b}$ * \\ aSAFRAN Paris Saclay, Rue des jeunes bois, Châteaufort CS 80112, 78772 Magny-les-Hameaux, France,
and b MINES ParisTech, PSL University, Centre des matériaux, CNRS UMR 7633, BP 87,91003 Evry Cedex,
France. Correspondence e-mail: henry.proudhon@mines-paristech.fr
}

A scanning laboratory Laue transmission setup is developed to probe extended quasi-monocrystalline samples. Orientation mapping is achieved by controlling the collimation of the incident beam and by scanning the position of the specimen. An automated indexing algorithm for transmission Laue patterns is presented together with a forward simulation model adapted for a laboratory setup. The effect of the main parameters composing the system is studied to aim for exposure time in the order of one second. Applications are presented to probe the orientation of an extended part and detect disoriented regions within the bulk. Finally the analysis of diffraction spot shapes showed that the misorientation within the illuminated volume can be measured and a new method is proposed to evaluate its complete mean lattice rotation tensor.
(C) 0000 International Union of Crystallography Printed in Singapore - all rights reserved
The general availability of electronic X-ray detectors and the emergence of third generation synchrotrons since the 1990s has radically changed how Laue experiments are performed. Submicron X-ray beam focusing, using a KB mirror placed before the sample (MacDowell et al., 2001), allowed polycrystalline samples to be studied by microLaue diffraction (Ice \& Larson, 2000). MicroLaue experiments on polycrystalline samples resulted in a considerable number of patterns acquired over a long period of time to achieve orientation and elastic strain mapping with micrometer resolution.

Several studies of Laue diffraction have also been carried out in laboratory conditions using latest X-ray source and detector technologies. A diffraction apparatus in side-reflection geometry named Laue Scanner, has been developed by Lehmann et al. (2014) to perform orientation mapping, which serves as an alternative to electron backscattered diffraction (EBSD) experiments. Similarly, a compact version of laboratory microLaue transmission diffraction has been proposed to determine grain orientations, and strain of polycrystalline sample (Lynch et al., 2007; Lynch et al., 2017; Lynch et al., 2019). The micron beam was obtained with the combination of a microfocus X-ray source and a monocapillary optic. One limitation of this setup is the maximum energy of $20 \mathrm{keV}$ which prevents the use of thick samples.

Studies in transmission conditions have also been carried out using a high energy Laue transmission setup by the LaueLangevin Institut in Grenoble (France) to perform Laue diffraction on massive monocrystalline samples (Bastie \& Hamelin, 1996; Hamelin et al., 2004). The facility used the X-ray diffraction focusing properties demonstrated by Guinier \& Tennevin (1949) with a high energy industrial source (from 100 to $400 \mathrm{kV}$ ) and a two-dimensional X-ray detector, both located at the same distance from the sample. The highest angular resolution of 10 seconds of arc for lattice rotation was obtained at 
6 meters of focusing distance, so that 12 meters separates the source from the detector. Although of great precision, this configuration requires a heavy and specific installation, which is not available in most laboratories and only captures individual diffraction spots.

The continued improvement of other X-ray sources and high dynamical X-ray detectors (Skarzynski, 2013) provides a renewed interest for Laue transmission diffraction methods in laboratory conditions. For instance, polychromatic diffraction contrast tomography (DCT) experiment can also be performed in laboratory conditions (King et al., 2014; Bachmann et al., 2019). Recently, a new technique called energy-dispersive Laue diffraction (EDLD) has been developed to determine the lattice parameters of an unknown crystal under synchrotron (Shokr et al., 2019) and laboratory (Kurdzesau, 2019) radiations. EDLD uses the latest generations of X-ray detector such as pnCCD (Leitenberger et al., 2008) or hybrid photon counting (HPC) detector (Brönnimann et al., 2001) to measure the energy of the diffraction spots.

One issue with the interpretation of the Laue patterns has always been to know the indices of the lattice planes involved with each diffraction spot, a process called indexing. Manual methods using charts and Wulff net (Cullity, 1956) have progressively been replaced by indexing programs (Preuss et al., 1974; Riquet \& Bonnet, 1979; Elder, 1986; Marin et al., 1994; Campbell et al., 1998). The first programs for indexing Laue diffraction pattern were written in FORTRAN and already allowed users to index diffraction patterns with minimum user interaction. Today, the large data sets, generally produced by side-reflection method under synchrotron radiation, are processed by new Laue software analysis method which includes image processing with peak search and indexing with tools such as LaueTools (Micha et al., 2012) or XMAS (Tamura, 2014).

Diffraction forward simulation frameworks have also been developed to generate virtual diffraction images using monochromatic high energy diffraction microscopy (HEDM) experiments (Wong et al., 2013). The sample is discretized in independent blocks to represent internal heterogeneities in a single crystal or grains in a polycrystal (Pagan \& Miller, 2014).

Measuring the lattice curvature is of great interest in particular within individual grains as it is a natural outcome of plastic deformation in polycrystal. For this reason, monochromatic synchrotron radiation studies are often employed while rotating the sample to measure individual grains rocking curves to access different component on the grain lattice rotation (Eberl et al., 2002). In some cases, it is possible to reconstruct the full orientation field of the grain (Simons et al., 2015). In modern experiments where the complete grain structure of the specimen is also measured, this can directly be compared with finite element predictions (Proudhon et al., 2018; Shade et al., 2019). Lattice rotation is also readily available from microLaue experiments. However, all of these methods require both a complicated synchrotron setup and a rotation (or beam scanning) of some kind of the sample.
Mechanical loading can also be coupled with Laue diffraction to provide in-situ tests, in side-reflection (Van Swygenhoven et al., 2006; Van Swygenhoven \& Van Petegem, 2013; Obstalecki et al., 2014) or in transmission modes (Hofmann et al., 2012a; Hofmann et al., 2012b). Because the reflected spot spreading can be related to the crystal lattice rotations (Calnan, 1952; Chung \& Ice, 1999; Barabash et al., 2003), the behaviour of a polycrystal material during mechanical testing can be studied using diffraction peak analysis. Spot spreading, also called asterism, has been related to the presence of geometrically necessary dislocations (Russell \& Ashby, 1970) that curve the crystal lattice (Barabash et al., 2001).

This work presents a compact laboratory high energy diffraction system, capable of working with several millimeters thick single crystal samples and has fast acquisition time. The goal is to evaluate the capability of the method for measuring the crystal orientation throughout a complete part via assessing the presence of non-desirable grain in single crystal or the presence of an orientation gradient. The setup is automated and utilizes recent industrial technologies for X-ray production and detection. Diffraction image analysis is performed by an in-house indexing algorithm, which take into account the specific geometry of the laboratory system and make use of a forward simulation model.

The paper is organized as follows: section 2 introduces all the geometry relations, presents the forward model, the indexing algorithm and a method to evaluate the mean crystal lattice curvature. Section 3 presents transmission Laue diffraction experiments, where the impact of the most important setup parameters is evaluated. Finally, section 4 shows applications of transmission Laue diffraction to detect and quantify variation from the perfect single crystal case in different samples.

\section{Laue transmission simulation and indexing}

\subsection{Forward simulation of transmission Laue diffraction}

Figure 1 depicts a schematic of a transmission Laue experiment, where a polychromatic X-ray beam strikes a crystalline sample placed in the laboratory coordinate system $\mathcal{R}_{l}(\mathrm{O}, \hat{\mathbf{X}}, \hat{\mathbf{Y}}, \hat{\mathbf{Z}})^{\mathbf{1}}$. In this work, the general case of a divergent beam limited by 4 motorized slits illuminating an extended sample is considered. Samples of interest are quasi-monocrystalline so that a main orientation can be defined. They may contain crystalline defects such as parasite grains or orientation gradient that we will assess by laboratory transmission Laue diffraction.

Following Bragg's law, a reflection $(h k l)$ may diffract for a particular wavelength $\lambda$ at an angle $\theta$ in the reflection plane defined by the incident beam $\hat{\mathbf{s}}_{0}$ and the scattering vector $\mathbf{q}$. $(h, k, l)$ are the Miller indices of the lattice plane. Proper Euler angles $\left(\varphi_{1}, \phi, \varphi_{2}\right)$ according to the Bunge convention, are used to parametrize the local orientation of the crystal. In the laboratory frame, the Bragg condition writes $\mathbf{G}_{l}=\mathbf{g}^{T} \mathbf{B} \mathbf{G}^{*}$, where $\mathbf{G}^{*}$ is the scattering vector in reciprocal space, $\mathbf{B}$ is the metric tensor and $\mathbf{g}$ is the orientation matrix. Detailed expressions for these quantities are widely available in the literature (Busing 
\& Levy, 1967; Poulsen, 2004). Note that the methodology presented here applies to any crystal lattice.

Laue transmission patterns are composed by discrete spots related to individual diffraction events. Diffraction spots are collected by a two dimensional X-ray detector, to which a Cartesian coordinate system $\mathcal{R}_{d}(\mathrm{D}, \hat{\mathbf{u}}, \hat{\mathbf{v}}, \hat{\mathbf{w}})$ is attached and is located in space by 6 degrees of freedom. The first 3 degrees of freedom define the position of $D$ the origin of the detector taken as the pixel of coordinates $(0,0)$ in the laboratory frame $\mathcal{R}_{l}$. The detector rotation can be defined by a series of 3 intrinsic rotations of the local frame attached to the detector. For simplicity here and without loss of generality, the detector is considered perfectly aligned in the plane (YZ) of the laboratory frame, so normal to $\hat{\mathbf{X}}$.

The simple model presented here works within the kinematical diffraction theory to generate Laue diffraction patterns based on Bragg's law and an extended sample geometry. The model, which can simulate any experimental configurations, will be later used by the indexing algorithm. It can also be used to study the impact of experimental parameters such as the source spectrum, the different working distances, the sample shape or the detector size. Python functions for the diffraction model have been integrated within the open source package pymicro ${ }^{2}$.

In the following, $\hat{\mathbf{s}}_{0}$ denotes the mean incident beam direction from $\mathrm{S}$, the X-ray source (considered punctual), to the central position of the slit aperture. The source provides a polychromatic radiation with a range of energy $\left[\mathrm{E}_{\min }, \mathrm{E}_{\max }\right] \mathrm{keV}$, placed in the laboratory frame at $\left(\mathrm{d}_{\mathrm{SX}}, \mathrm{d}_{\mathrm{SY}}, \mathrm{d}_{\mathrm{SZ}}\right)$ (see Fig. 1). $\hat{\mathbf{s}}_{0}$ direction can be adequately parametrized by two combined rotations first using angle $\xi_{u}$ in the (XY) plane and then angle $\xi_{v}$ in (ZX) plane. $\hat{\mathbf{s}}_{0}$ is defined as:

$$
\hat{\mathbf{s}}_{0}=\left\{\begin{array}{c}
\cos \xi_{u} \cos \xi_{v} \\
\sin \xi_{u} \cos \xi_{v} \\
-\sin \xi_{v}
\end{array}\right\}
$$

Punctual diffraction simulation is applied to a virtual sample reduced at a point at the origin of the $\mathcal{R}_{l}$ frame, which will also be the origin of each diffraction event. The sample is described using the Bravais lattice $(a, b, c, \alpha, \beta, \gamma)$ of a material and the lattice orientation $\left(\varphi_{1}, \phi, \varphi_{2}\right)$ to generate the reciprocallattice frame $\mathcal{R}^{*}$. The forward simulation requires a set of lattice planes $(h k l)$ which is limited by the maximum value of the Miller indices considered. To limit the number of lattice planes, only first order reflections are kept, as higher orders have been shown to superimpose in Laue diffraction (Amorós et al., 1956).

Bragg angle $\theta$ and associated wavelength $\lambda$ are then calculated for each reflection in condition of diffraction with:

$$
\begin{aligned}
\theta & =\arccos \left(\hat{\mathbf{s}}_{0} \cdot \hat{\mathbf{G}}_{l}\right)-\frac{\pi}{2} \\
\lambda & =2 d_{h k l} \sin \theta
\end{aligned}
$$

where $d_{h k l}$ is the interplanar spacing of lattice planes of indices $(h, k, l)$. If the wavelength range of the source $\left[\lambda_{\min }, \lambda_{\max }\right]$ is considered, the diffracting event can be discarded if $\lambda$ lies outside of it. The correspondence between wavelength and energy is given by: $\mathrm{E}(\mathrm{keV})=1.2398 / \lambda(\mathrm{nm})$ (Als-Nielsen \& McMorrow, 2011).

For the given wavelength, the scattering vector is linked to the difference between the incident beam $\hat{\mathbf{s}}_{0}$ and the reflected beam $\hat{\mathbf{s}}$ (Warren, 1969):

$$
\mathbf{q}=\frac{\hat{\mathbf{s}}-\hat{\mathbf{s}}_{0}}{\lambda}
$$

Laue diffraction occurs when the scattering vector coincides with a reciprocal-space lattice vector which here is implied by Eqs. $(2$ - 3). Finally, the diffracted vector is written in the laboratory frame by:

$$
\hat{\mathbf{s}}=\hat{\mathbf{s}}_{0}+\lambda \mathbf{G}_{l}
$$

The detector is represented by a regular grid of pixels of a given size in the detector frame $\mathcal{R}_{d}$. Each reflection $\mathbf{s}$ intersects the detector plane at a position denoted by $(\mathrm{u}, \mathrm{v})$. If $(\mathrm{u}, \mathrm{v})$ falls outside the detector grid, the diffraction event is discarded. An intensity has been assigned to the pixels identified to create a punctual diffraction simulation pattern.

This simple point diffraction forward model can also be used to simulate an extended sample geometry, which corresponds to the volume illuminated by the incident beam. This geometry is discretized using a grid of isotropic voxels located in $\mathcal{R}_{l}$ coordinate system, see Fig. 2. In this case, each voxel $i$ is located at position $\mathrm{V}_{i}(\mathrm{x}, \mathrm{y}, \mathrm{z})$ and for each position, the incident wave vector $\mathbf{s}_{0 i}$ is calculated from the source $\mathrm{S}$. Eq. (5) can be now applied for each voxel composing the sample using the specific incident wave vector $\mathbf{s}_{0 i}$. Simulated diffraction spots are now composed of as many $\mathbf{s}_{i}$ reflections as there are voxels. The forward simulation of a diffraction pattern for an extended sample is performed by incrementing the pixel intensity for each $\mathbf{s}_{i}$ projection onto the detector. For simplicity, the diffracted intensity is not taken into account and as a result the simulated intensity is only a function of the crystal shape and of the lattice rotation distribution. Fig. 3 presents examples of forward simulation diffraction pattern for an extended sample and shows that for a single orientation the finite size of the sample enlarges the diffraction spots. Note that to display diffraction patterns, an inverse gray scale colormap is used where spots appear in dark over a white background. It also shows that the Laue focusing (Guinier \& Tennevin, 1949) is naturally reproduced in the plane of each reflection. This is modified if a gradient of orientation is present as we shall see in more details in Section 4.3. In addition, with the finite size of the sample, a single spot is no longer monochromatic and is now composed of a energy range (typically several $\mathrm{keV}$ ). Increasing the aperture size i.e. the size of the illumination in the (YZ) plane, increases the variation of the $\mathrm{X}$-ray incident angle which in turn, increases the energy range for a given spot.

\subsection{Indexing algorithm}

Laue pattern indexing can be performed in several ways (Cullity, 1956; Wenk et al., 1997; Tamura, 2014). A direct indexing method has been chosen here and the logic is shown in Fig. 4. 
The inputs of the algorithm are the $(u, v)$ coordinates of each reflection spot, the lattice parameters of the crystal and the acquisition geometry that comes from a setup calibration (see later in Sec. 3.2). The spot coordinates (intensity weighted average) are automatically obtained by a peak search function. From there, Eq. (5) can be re-written for each reflection $k$ to obtain its reflection normals $\mathbf{N}_{k}$ :

$$
\mathbf{N}_{k}=\lambda_{k} \mathbf{G}_{k, l}=\hat{\mathbf{s}}_{k}-\hat{\mathbf{s}}_{0}
$$

By determining the lattice plane normal directions, the angles between all pairs of directions can be calculated. Experimental angles are stored in a dedicated table, called Experimental angles table which is compared with the Theoretical angles table that contains angles calculated from a generated list of $(h \mathrm{kl})$ planes. Angles are compared to each other within a fixed tolerance (here $0.5^{\circ}$ ) using triplets of spots to reduce the number of possible solutions. With identified $(h k l)$ triplets, a corresponding rotation matrix can be constructed by generating two frames named $\mathcal{E}^{*}$ and $\mathcal{E}_{c}$ (Wright $\&$ Adams, 1992). The frame $\mathcal{E}^{*}=\left(\hat{\mathbf{e}}_{1}^{*}, \hat{\mathbf{e}}_{2}^{*}, \hat{\mathbf{e}}_{3}^{*}\right)$ is constructed from 2 normal directions $\mathbf{N}_{i}$ and $\mathbf{N}_{j}$ from the triplet such as:

$$
\begin{gathered}
\hat{\mathbf{n}}_{i}^{*}=\frac{\mathbf{N}_{i}}{\left|\mathbf{N}_{i}\right|}, \hat{\mathbf{n}}_{j}^{*}=\frac{\mathbf{N}_{j}}{\left|\mathbf{N}_{j}\right|} \\
\hat{\mathbf{e}}_{1}^{*}=\hat{\mathbf{n}}_{i}^{*}, \hat{\mathbf{e}}_{2}^{*}=\frac{\hat{\mathbf{n}}_{i}^{*} \wedge \hat{\mathbf{n}}_{j}^{*}}{\left|\hat{\mathbf{n}}_{i}^{*}\right| \cdot\left|\hat{\mathbf{n}}_{j}^{*}\right|}, \hat{\mathbf{e}}_{3}^{*}=\hat{\mathbf{e}}_{1}^{*} \wedge \hat{\mathbf{e}}_{2}^{*}
\end{gathered}
$$

The second frame $\mathcal{E}_{c}=\left(\hat{\mathbf{e}}_{c_{1}}, \hat{\mathbf{e}}_{c_{2}}, \hat{\mathbf{e}}_{c_{3}}\right)$ is constructed using the two sets of Miller indices $(h k l)_{i}$ and $(h k l)_{j}$ identified for $\mathbf{N}_{i}$ and $\mathbf{N}_{j}$ :

$$
\begin{gathered}
\hat{\mathbf{n}}_{i}=\frac{\mathbf{n}_{h k l_{i}}}{\left|\mathbf{n}_{h k l_{i}}\right|}, \hat{\mathbf{n}}_{j}=\frac{\mathbf{n}_{h k l_{j}}}{\mid \mathbf{n}_{h k l_{j} \mid}} \\
\hat{\mathbf{e}}_{c_{1}}=\hat{\mathbf{n}}_{i}, \hat{\mathbf{e}}_{c_{2}}=\frac{\hat{\mathbf{n}}_{i} \wedge \hat{\mathbf{n}}_{j}}{\left|\hat{\mathbf{n}}_{i}\right| \cdot\left|\hat{\mathbf{n}}_{j}\right|}, \hat{\mathbf{e}}_{c_{3}}=\hat{\mathbf{e}}_{c_{1}} \wedge \hat{\mathbf{e}}_{c_{2}}
\end{gathered}
$$

Finally, the corresponding rotation matrix for the couple of indexed spots $(i, j)$ is obtained by:

$$
\mathbf{g}^{i j}=\mathbf{e}_{c}^{t} \cdot \mathbf{e}^{*}
$$

Here, the symmetry of the crystal lattice needs to be taken into account. To avoid the indexing algorithm treating symmetrically equivalent rotations ( 24 for the cubic lattice studied hereafter) as different solutions, all $\mathbf{g}^{i j}$ are moved to the Rodrigues fundamental zone and the rotation involving the minimum angle is selected (Karthikeyan et al., 2012).

As a $\mathbf{g}_{i j}$ matrix is calculated for each indexed triplet, the indexing algorithm must select a unique solution. This final step is performed by a poll system where each possible solution is stored and ranked by the number of time it appears in the algorithm (a tolerance based on misorientation is used here). The orientation with first rank is defined as the solution $\mathbf{g}_{\mathrm{sol}}$.

\subsection{Measuring mean crystal lattice curvature}

In this section, a simple method to determine the mean lattice curvature tensor $\underset{\sim}{\kappa}$ (Nye, 1953) from a Laue diffraction image is presented. $\boldsymbol{\kappa}$ is a second order tensor represented by a $3 \times 3$ matrix in the crystal frame $\mathcal{R}_{c}$ which can also be expressed in the laboratory frame by

$$
\underset{\sim}{\boldsymbol{\kappa}_{l}}=\mathbf{g}_{r e f}^{T} \underset{\sim}{\boldsymbol{\kappa}_{c}} \mathbf{g}_{r e f}
$$

where $\mathbf{g}_{\text {ref }}$ represents the reference orientation of the crystal. Let $\phi(\mathrm{x}, \mathrm{y}, \mathrm{z})$ be the rotation field describing the change of orientation of a material point located at $(x, y, z)$ with respect to the reference orientation. $\underset{\sim}{\kappa}$ is defined as the derivative of $\phi$ so that:

$$
\mathrm{d} \phi=\underset{\sim}{\boldsymbol{\sim}_{l}} \cdot \mathrm{dX}
$$

The corresponding rotation matrix $\mathbf{g}_{\kappa}$ is calculated and used to obtain the material point orientation matrix:

$$
\mathbf{g}(\mathrm{x}, \mathrm{y}, \mathrm{z})=\mathbf{g}_{r e f} \cdot \mathbf{g}_{\kappa}(\mathrm{x}, \mathrm{y}, \mathrm{z})
$$

The scattering vector at location $(x, y, z)$ is expressed as :

$$
\begin{aligned}
\mathbf{G}_{l}(x, y, z) & =\left(\mathbf{g}_{r e f} \mathbf{g}_{\kappa}\right)^{-1} \mathbf{G}^{*} \\
& =\mathbf{g}_{\kappa}^{-1} \mathbf{g}_{r e f}^{-1} \mathbf{G}^{*} \\
& =\mathbf{g}_{\kappa}^{-1}(x, y, z) \mathbf{G}_{l, r e f}
\end{aligned}
$$

Finally the diffracted vector at this point is:

$$
\hat{\mathbf{s}}(x, y, z)=\hat{\mathbf{s}}_{0}+\lambda \mathbf{g}_{\kappa}^{-1}(x, y, z) \mathbf{G}_{l, r e f}
$$

Now, assuming the lattice curvature can be described by a mean $\underset{\sim}{\kappa}$ over the illuminated volume, Eq. (14) shows that the extremities of the diffraction spot will carry information about $\underset{\sim}{\kappa}$ sampled by vector $\mathrm{dX}$ in Eq. (11). Since each spot will give some information in a different direction, the combination of the results for all spots should allow for complete evaluation of the lattice curvature.

This hypothesis has been tested on a discretized sample (see Fig. 2) where a rotation field is applied at each voxel regarding its position in the volume using a constant lattice curvature. Figure 5 shows the result of a comparison with and without lattice curvature to show the impact on the spot spreading for an illuminated volume of $1 \times 1 \times 1 \mathrm{~mm}^{3}$ discretized into $31^{3}$ voxels. Forward simulation is carried out on a Nvidia Quadro P4000 $\mathrm{GPu}$ card to speed up computation time, which then reduces it to a couple of seconds. This simulation example has been performed with the acquisition parameters of the nickel sample presented later (see Fig. 10) and calculation has been reduced at a point to eliminate sample volume effect. This example uses the following value for $\aleph_{c}$ :

$$
\underset{\sim}{\boldsymbol{\kappa}_{c}}=\left(\begin{array}{ccc}
2^{\circ} & 0^{\circ} & 0^{\circ} \\
0.5^{\circ} & 0.8^{\circ} & 0^{\circ} \\
0.25^{\circ} & 0^{\circ} & -0.5^{\circ}
\end{array}\right) \times \frac{\pi}{180^{\circ}} \times 10^{-3}[\mathrm{rad} / \mathrm{mm}]
$$

The change in orientation at a given point will affect the diffracting conditions and cause spread in the spot shape (asterism) which can be measured on the images. Eqs. (11-12) show that provided the illuminated volume is known (i.e. $\mathrm{dX}$ can be estimated), the measure of the spread can be used to retrieve $\underset{\sim}{\kappa}$. The estimation of the 9 components of the curvature tensor $\underset{\sim}{\kappa}$ represents the curvature around and following the 3 frame directions, 
thus the model requires the acquisition of 3 diffraction patterns with different illumination conditions (so to access 3 different $\mathrm{dX}$ vectors in Eq. (11)).

The spot shape analysis consists of selecting the extreme pixels on each spot and relating them to the 2 positions in the sample separated by $\mathrm{dX}$. In the case of a parallelepipedic illuminated volume, the extreme points are 2 of the 8 vertices of the volume (see Fig. 5 for an example of a cube). The normals for these 2 pixels are then calculated to obtain the 2 extreme $\mathbf{G}_{l}$ for each $(h k l)$ plane and for the 3 different patterns. The estimation of $\underset{\sim}{\boldsymbol{\kappa}}$ is performed by a least square optimization which allows to find the lattice curvature from which the forward model will best fit all measured $\mathbf{G}_{l}$.

This minimization allows us, in this rather perfect case of forward simulated images, to find the initial $\boldsymbol{\sim}_{c}$ (see Eq. (15)) with the precision of one tenth of a degree. This algorithm has yet to be tested on real experimental data. Several issues need to be taken into account: the illuminated volume needs to be precisely known to use the corresponding $\mathrm{dX}$ vectors; this finite volume will be convoluted to the lattice curvature to produce the spread of diffraction spot. Nonetheless, the full mean lattice curvature tensor can be recovered with only three diffraction patterns obtained by changing the aperture of the slits. This provides an elegant way to study the inner lattice curvature of a single crystal part.

\section{Diffraction system}

\subsection{Transmission Laue diffraction experimental setup}

The Laue diffraction setup uses a tunable X-ray tube, an Xray detector (preferably mobile), and an in-house portable stage featuring an X-ray motorized slits, a mobile sample support and a beam stop (see Fig. 6). Laue transmission diffraction experiments can be performed with any well-sized X-ray system with a sufficiently high energy tube $(>100 \mathrm{kV})$ and low dark noise detector. The energy of the X-ray tube allow to work with a thick sample $(>1 \mathrm{~mm}$ ) using the transmission method can be estimated by the Beer-Lambert law. The position of the detector should be adjusted to optimize the diffraction spot number and visibility. This usually means bringing the detector close to the sample as moving it away will reduce the angle range and increase absorption of diffracted beams by air significantly for a distance in the order of $1 \mathrm{~m}$. With the present sample stage, the detector can be placed as close as $10 \mathrm{~cm}$ from the sample. If the X-ray source is placed upstream at the same distance, the Laue focusing geometry (Guinier \& Tennevin, 1949) is achieved which increases the spot contrast. A good diffraction image is a compromise between the size of the detector and the number of spots required for indexing while keeping the exposure time in the order of the second, the general target in this work.

Several X-ray systems have been tested in this work. One of them is a tomographic platform (RX Solutions) located at the Navier laboratory, ENPC, France. It is operated with a $230 \mathrm{kV}$ open type microfocus HAMAMATSU L10801 X-ray tube and a VARIAN PaxScan ${ }^{\circledR} 2520$ V flat panel imager $(1920 \times 1560$ pixels with a size of $127 \mu \mathrm{m})$, see Fig. 7a. Another X-ray system, located at DECTRIS (Switzerland), comprises of a GE ISOVOLT Mobile 160 M1/10-55 X-ray tube and a DECTRIS PILATUS3 X CdTe HPC detector $(487 \times 619$ pixels with a size of $172 \mu \mathrm{m})$, see Fig. $7 \mathrm{~b}$.

The large conical beam provided by X-ray tubes is limited by motorized air slits (from JJ X-ray) with a controllable aperture in both $\mathrm{Y}$ and $\mathrm{Z}$ directions. The positioning of the sample is achieved by 2 motorized translations. Finally, the beam stop has been designed to be mobile in the three directions to simplify its alignment with respect to the transmitted incident beam.

\subsection{Setup calibration}

A precise calibration of the diffraction setup geometry is essential for the indexing algorithm to work properly (see Sec. 2.2). As the stage is portable, this step has to be repeated for each experiment. A reference silicon single crystal plate with a thickness of $0.5 \mathrm{~mm}$ has been chosen to calibrate the geometry. The orientation of this reference crystal, positioned on a dedicated support sample is $\left(\varphi_{1}=0^{\circ}, \phi=45^{\circ}, \varphi_{2}=0^{\circ}\right)$.

The determination of the setup geometric parameters, i.e. angles $\left(\xi_{u}, \xi_{v}\right)$, and distance $\mathrm{d}_{\mathrm{X}}$, is done using the forward model presented in Section 2.1. The parameters are refined using a minimization of the Euclidean distance between the reflected and the simulated spots.

$$
\begin{gathered}
\chi=\left(\varphi_{1}, \phi, \varphi_{2}, \xi_{u}, \xi_{v}, \mathrm{~d}_{\mathrm{X}}\right) \\
f(\chi)=\sum_{i} \sqrt{\left(\mathrm{u}_{\exp _{i}}-\mathrm{u}_{\operatorname{sim}_{i}}(\chi)\right)^{2}+\left(\mathrm{v}_{\exp _{i}}-\mathrm{v}_{\operatorname{sim}_{i}}(\chi)\right)^{2}}
\end{gathered}
$$

with $\left(\mathrm{u}_{\text {exp }}, \mathrm{v}_{\text {exp }}\right)$ and $\left(\mathrm{u}_{\text {sim }}, \mathrm{v}_{\text {sim }}\right)$ representing the positions on the detector frame of the experimental and simulated spots respectively. The optimization process may also include detector tilts if necessary (omitted here for simplicity).

\subsection{Experimental acquisition parameters}

Several Laue transmission diffraction experiments have been performed to characterise the impact of the acquisition parameters of a laboratory device.

Size of slits aperture The X-ray tube produces an incident cone that is then resized and directed by the slits collimator where the 4 slits can be operated independently, see Fig. 8. For a fixed sample position, the direction and the divergence of the beam are directly linked with the diffraction pattern.

For a given source-sample distance, the incident beam divergence is controlled by the size of the slits aperture. Most Laue reflection experiments use a highly collimated beam while here the laboratory transmission setup utilizes a much more divergent beam with a tested aperture up to $12 \mathrm{~mm}^{2}$, which results in the illumination of a large volume of the sample (see Fig. 8 Case b.). Patterns shown on Fig. 9 have been acquired with a $1 \mathrm{~mm}$ thick of nickel-based alloy, not in focusing condition, to study the impact of the divergence of the spots shape. Where a small aperture of $1 \times 1 \mathrm{~mm}^{2}$ generates rounded spots, an enlarged aperture of $4 \times 3 \mathrm{~mm}^{2}$ creates enlarged spots in the direction 
normal to the reflection plane (the size corresponds to the aperture in this direction). The height of the spots in the reflection plane is related to the setup geometry with the distances $d_{X}$ and $\mathrm{d}_{\mathrm{SX}}$, and is not influenced by the size of the aperture in the perpendicular direction (in first approximation). In reality this is convoluted with the presence of lattice rotations within the illuminated volume that modify the size and shape of the spots on the detector (see Sec. 2.3). The forward simulation of both illuminated volumes of $1 \mathrm{~mm}^{3}$ and $12 \mathrm{~mm}^{3}$ allows to compare the energy range involved on spots. The large volume of illuminated sample combined with the divergence of the beam generates diffracted beams that contain a large range of energy, up to $35 \mathrm{keV}$ for planes $(\overline{3} 5 \overline{1})$ and $(220)$, where the energy ranges involved are respectively $[79,114] \mathrm{keV}$ and $[50,85] \mathrm{keV}$. The enlarged aperture of $4 \times 3 \mathrm{~mm}^{2}$ also significantly reduces the acquisition time since a unique image performed at $1 \mathrm{~s}$ of exposure gives equivalent information as 12 images of $1 \times 1 \mathrm{~mm}^{2}$ aperture.

Slits position The mean incident beam direction is controlled by the position in space of the center of the slits (see Fig. 8 Case a. and c.). To study the impact of this parameter, let's suppose a small aperture of the slits assimilable to a pencil beam. This direction, represented by $\hat{\mathbf{s}}_{0}$, is used in Eq. (5) to compute the reflected beam direction, which involves a modification of the diffraction pattern in case of a change in $\hat{\mathbf{s}}_{0}$. Examples of patterns with different incident beam directions obtained by scanning the slits in the $\mathbf{Y}$ direction are presented on Fig. 10. This experiment has been performed on a $1 \mathrm{~mm} \times 1 \mathrm{~mm} \times$ $10 \mathrm{~mm}$ nickel bar, positioned vertically in the support sample. A series of 7 patterns have been acquired for different $\xi_{u}$ angle, from $2.78^{\circ}$ to $-1.81^{\circ}$ and $\xi_{v}$ angle sets at $1.21^{\circ}\left(0.03^{\circ}\right.$ of standard deviation). Note that the sample is translated horizontally so that the illuminated volume stays the same for each pattern. Patterns show movements of the spots positions, including some spots appearing or disappearing. Spots diffracting energies have been modified by the change in $\hat{\mathbf{s}}_{0}$ : planes $(1 \overline{1} \overline{1})$ and $(\overline{1} 1 \overline{1})$ were diffracting respectively at $36 \mathrm{keV}$ and $34 \mathrm{keV}$ with $\xi_{u}=2.3^{\circ}$ and moved to $27 \mathrm{keV}$ and $48 \mathrm{keV}$ with $\xi_{u}=0.44^{\circ}$.

The indexing has been carried out arbitrarily on the pattern showing the higher symmetry (Fig. 10 - Pos. 2). As the crystal orientation is fixed during the sample displacement, the 6 other patterns have been indexed using a refinement (see Eq. (16)) based on $\chi=\left(\xi_{u}, \xi_{v}, \mathrm{Ox}, \mathrm{Oy}, \mathrm{Oz}\right)$, where $(\mathrm{Ox}, \mathrm{Oy}, \mathrm{Oz})_{\mathcal{R}_{l}}$ are the coordinates of the origin diffracting point position. The graph shown in Fig. 10 displays the obtained sample position in the (YZ) plane. This shows how indexing the diffraction pattern allow to obtain the position of the illuminated volume in the laboratory frame.

X-ray detector technology An experimental diffraction pattern also highly depends on the X-ray detector geometry and capability. Two types of detector have been used in this study (see Sec. 3.1): a flat panel and an HPC detector. Fig. 11 shows Laue transmission patterns of $1 \mathrm{~mm}$ thick nickel-based alloy acquired with different acquisition parameters and the DEC-
TRIS PILATUS3 X CdTe detector. With the fast acquisition time of this detector, only $0.1 \mathrm{~s}$ is needed to obtain a sufficiently good signal to noise ratio and enough spots to be able to use the indexing algorithm (see Fig. 11a). Figure 11b presents a diffraction pattern without any beam-stop where the transmitted beam contains 1500 times more photons than in reflected spots without any saturation or damage. The high dynamical range of the PILATUS3 X CdTe detector is demonstrated, which when combined with its low noise, makes this detector highly desirable in these kind of experiments to detect both intense and weak reflections.

Not using a beam-stop simplifies the setup. However, the high contrast difference between the transmitted beam with its diffusion halo and the reflection spots make the peak search more complex. The peak search accuracy is essential for correct indexing, which still makes the use of a beam stop desirable.

\section{Quantitative evaluation of crystalline quality}

\subsection{Orientation mapping}

Using the indexing of Laue diffraction patterns, orientation mapping of an extended part can be achieved by scanning the sample perpendicularly to the X-ray beam to collect diffraction patterns from the complete volume. In the present work, the sample can be moved in front of the incident beam by the two translation stages (see Sec. 3.1 and Fig. 6). This scanning reconstruction can reveal the presence of a local disoriented volume of material which can be quantified by its size and the misorientation with respect to the reference orientation.

Figure 12 shows an example of a diffraction pattern, where the local disoriented volume is smaller than the illuminated one. In this case, the spot shapes are directly convoluted with the size of the disoriented crystal in the volume. Indexing correctly such a small disoriented crystal necessitate a sufficient number of spots. In this special case, the current experimental setup permits the location of the disoriented grain following the two scanning directions $\mathbf{Y}$ and $\mathbf{Z}$. The determination of the position of this grain along the $\mathbf{X}$ direction may only be approximately achieved by optimizing the object to detector distance after indexing.

In the case of the presence of a large disoriented crystal, the scan of the sample shows the transition between both grains with the progressive modification of the spot positions, see Fig. 13. This experiment has been performed with a $1 \mathrm{~mm}$ thick nickel-based sample. Diffraction patterns are captured from an illuminated volume of $1 \mathrm{~mm}^{3}$ and the scan step is $1 \mathrm{~mm}$ along the $\mathbf{Y}$ axis. After indexing, the misorientation between the two grains is calculated to be $10.1^{\circ}$. A relatively precise definition of the grain boundary location can be obtained by reducing the incident beam divergence although the information is averaged in the $\mathbf{X}$ direction (the sample thickness).

Orientation mapping has been carried out on a tri-crystalline nickel-based alloy plate shape sample with an average thickness of $1.5 \mathrm{~mm}$. The size of the incident beam was about $1.5 \times 1.5 \mathrm{~mm}^{2}$. A rectangular grid of 72 patterns has been collected from an area of $7 \times 5 \mathrm{~mm}^{2}$. The final mapping is presented 
Fig. 14, where the three grains (denoted A, B, and C) have been located in the sample. The mean misorientation between the grain $\mathrm{A}$ and $\mathrm{B}$ is $7.8^{\circ}$. For simplicity, grain $\mathrm{C}$ has only been detected and misorientation was not determined (given its size, grain $\mathrm{C}$ always appear combined with grain $\mathrm{B}$ which did not allow automated indexing).

The result of indexing the 72 patterns is shown on the inverse pole figure in the unit standard triangle. The inverse pole figure indicates that grain A is composed of 3 sub-grains disoriented one from another about $0.6^{\circ}, 1.1^{\circ}$ and $0.9^{\circ}$, where "subgrain" implies the conservation of the Bragg reflections despite the rotation.

\subsection{Microstructure within the illuminated volume}

Several experimental diffraction patterns of nickel-based alloy have presented spots composed of individual sub-spots. Figure 15 provides an enlargement of 3 reflections where the sub-spot coordinates have been manually clustered. From there, the sub-indexing has been carried out using a refinement (see Eq. (16)) based on $\chi=\left(\varphi_{1}, \phi, \varphi_{2}, \mathrm{Ox}, \mathrm{Oy}, \mathrm{Oz}\right)$ : the crystal orientation and the origin of the diffracting point within the illuminated volume, as the sub-spots come from sub-orientations. The result of the refinement shows that misorientations between the 3 sub-grains within the illuminated volume are $0.5^{\circ}$ (bluegreen), $0.3^{\circ}$ (red-blue) and $0.6^{\circ}$ (red-green).

Then, the diffracting point origins can be used to estimate the microstructure (i.e. the mean position of the sub-grains) inside the illuminated volume. Using the intensity ratios of the subspots, a Laguerre tessellation is applied to recover the proportion of sub-grains within the volume and obtain an approximation of the microstructure in 3 dimensions (see Fig. 15).

\subsection{Inner crystal lattice curvature}

Figure 16 depicts a series of diffraction patterns which have been acquired in the same focusing condition of $d_{S X}=d_{X}=$ $125 \mathrm{~mm}$ with fixed acquisition parameters for the X-ray tube and detector which shows large differences in terms of spot shapes. The Laue focusing condition, concentrates reflected beams of the incident divergent beam on a given point in the reflection plane but the size in the direction normal to the reflection plane remain proportional to the slits aperture in that direction. The very thin (4 pixels thickness) rod shape in Fig. 16(a) and (b) of both silicon and germanium samples, are the results of an almost perfect beam focusing (the remaining spot width can be primarily attributed to the effect of the sample thickness as shown by a forward simulation). Despite this focusing condition, Fig. 16(c) and (d) of pure nickel and nickel-based alloy samples present much rounder shaped spots which implies deviations from the case of a perfect crystal lattice. Assuming no residual stress is present, the rounded spots are the signature of lattice rotations present within the illuminated volume. Using, for simplicity, the spot diffracting close to the (XZ) plane in Fig. 16(d) give a spread of $1.9 \mathrm{~mm}$ average (corrected from sample thickness broadening using the forward model) which correspond to a $0.7^{\circ} \pm 0.1$ deviation from the main Bragg condition. As the illuminated volume is $1 \mathrm{~mm}^{3}$, this is a measure of the curvature around the $\mathbf{Y}$ axis.

To verify this, the lattice curvature has also been evaluated by EBSD in the same region at the sample surface which shown an orientation gradient of $0.8 \% \mathrm{~mm}$ around the same axis. As shown in Section 2.3, using all the spot and 3 patterns acquired with 3 different apertures should allow to compute the full mean lattice curvature tensor. In contrast, the silicon and germanium samples do not show any broadening, which means they contain no measurable crystal lattice rotation and can be evaluated as perfect crystals. This shows that in addition to measuring the crystalline orientation, this laboratory transmission diffraction setup can be used to quickly probe the crystalline quality.

\section{Discussion}

The present results combining a compact setup, a general indexing method and a forward model, show the interest of the high energy Laue transmission diffraction method to evaluate single crystal quality. The dedicated setup has demonstrated its efficiency regardless the X-ray system on a number of configuration to probe crystal orientation, detect parasite grains, perform scanning orientation mapping and measure lattice curvature within the illuminated volume. Regarding working distances, 10 to $15 \mathrm{~cm}$ appears as a good compromise when combined with state of the art 2D detector size of 10 to $30 \mathrm{~cm}$. The compact design of our setup allows Laue focusing geometry with an important angular range available to collect Bragg reflections. A $160 \mathrm{kV}$ high energy X-ray source appeared to be adequate to collect a significant number of reflections through up to a few mm of nickel-based alloy, even more in a less absorbing material. In this regard, this method remain limited to samples or parts not exceeding this thickness. To give an idea of the detection limit, a grain of $1 \times 2 \times 0.3 \mathrm{~mm}^{3}$ has been detected through $5 \mathrm{~mm}$ of nickel based alloy, our actual best performance, using a HPC detector. This type of imaging is indeed ideal for this application, the photon counting accuracy, the high dynamical range and the absence of dark noise allowing fast and effective acquisitions. The relatively large size of this type of detector being an additional benefit to capture a large angular range. The impact of the X-ray source spot size has not been evaluated yet but appear to be negligible regarding the good correlation of the forward model and the experiments without taking it into account. Future improvements could be to add a more general 6 degrees of freedom robotic arm to place and orient the sample in front of the beam. Improvements of the forward model should include modelling the spot intensities and using a realistic source spectrum (Dejoie et al., 2011).

Laue transmission diffraction could be used for nondestructive testing applications. The manufacture of single crystal for industrial applications such as for turbine blades, can involve local crystalline disorientation in the bulk. Although this requires to deal with complex geometry (typically the 2 walls of the blade split each diffraction spot in 2), non-desirable local disorientation can in principle be detected in the volume by this technique. In this case, the use of a forward model taking into account the precise geometry of the part and of the system will be a real advantage. As shown in the previous examples, 
the detection capability of such a diffraction setup will depend on the specimen thickness, the divergence of the incident beam and on the scanning step size.

\section{Conclusion}

Technological performances of X-ray sources and recent detectors renew the interest in a laboratory system for Laue transmission diffraction. While this type of system can be used as a classical Laue device, that is, to measure crystal lattice orientation of a sample, other applications have been demonstrated including the determination of the crystal curvature and the orientation mapping of an entire part. This application can not only be useful in laboratory research environment, but also in industry settings for non-destructive test of entire parts with a thickness up to several $\mathrm{mm}$.

Acknowledgements The authors are grateful to SAFRAN for the funding of this study. We thank Patrick Aimedieu and Michel Bornert, researchers at Navier laboratory for their great help operating the tomographic platform. We thank also the workshop of the Centre des Matériaux, l'Atelier JPE, for all advises and help with mechanical realisations. We thank as well Vincent Revol for his help at CSEM X-ray facility and Nicolas Pilet and Dubravka Sisak Jung for their helps at DECTRIS Xray facility. Henry Proudhon thanks Samuel Forest for the fruitful discussion on the lattice curvature tensor. Finally, we want to thank the ANRT for subsiding this PhD program $N^{\circ} 2016 / 0993$.

\section{References}

Als-Nielsen, J. \& McMorrow, D. (2011). Elements of modern X-ray physics. John Wiley \& Sons.

Amorós, J.-L., Buerger, M.-J. \& de Amorós, M.-C. (1956). The Laue Method. Academic Press.

Bachmann, F., Bale, H., Gueninchault, N., Holzner, C. \& Lauridsen, E. M. (2019). Journal of Applied Crystallography, 52(3).

Barabash, R., Ice, G., Larson, B., Pharr, G., Chung, K.-S. \& Yang, W. (2001). Applied Physics Letters, 79(6), 749-751.

Barabash, R., Ice, G. \& Walker, F. (2003). Journal of applied physics, 93(3), 1457-1464.

Bastie, P. \& Hamelin, B. (1996). Journal de Physique IV, 6(C4), C413.

Brönnimann, C., Baur, R., Eikenberry, E., Kohout, S., Lindner, M., Schmitt, B. \& Horisberger, R. (2001). Nuclear Instruments and Methods in Physics Research Section A, 465(1), 235-239.

Busing, W. R. \& Levy, H. A. (1967). Acta Crystallographica, 22(4), 457-464.

Calnan, E. (1952). Acta Crystallographica, 5(5), 557-563.

Campbell, J. W., Hao, Q., Harding, M. M., Nguti, N. D. \& Wilkinson, C. (1998). Journal of Applied Crystallography, 31(3), 496-502.

Chung, J.-S. \& Ice, G. E. (1999). Journal of applied physics, 86(9), $5249-5255$.

Cullity, B.-D. (1956). Elements of X-ray Diffraction. Addison-Wesley.

Dejoie, C., Kunz, M., Tamura, N., Bousige, C., Chen, K., Teat, S., Beavers, C. \& Baerlocher, C. (2011). Journal of Applied Crystallography, 44(1), 177-183.

Eberl, F., Forest, S., Cailletaud, G., Wroblewski, T. \& Lebrun, J. L. (2002). Metallurgical and Materials Transactions A, 33(9), $2825-2833$.

Eckert, M. (2012). Annalen der Physik, 524(5), A83-A85.

Elder, M. (1986). Information Quarterly for Protein Crystallography, 15(19), 31.

Geisler, A. \& Hill, J. (1948). Acta Crystallographica, 1(5), 238-252.

Greninger, A. B. (1935). Zeitschrift für Kristallographie-Crystalline Materials, 91(1-6), 424-432.
Guinier, A. \& Tennevin, J. (1949). Acta Crystallographica, 2(3), 133138.

Hamelin, B., Bastie, P., Richard, D. \& Eiaazzouzi, A. (2004). Journal de Physique IV, 118(1), 437-446.

Helliwell, J., Habash, J., Cruickshank, D., Harding, M., Greenhough, T., Campbell, J., Clifton, I., Elder, M., Machin, P., Papiz, M. et al. (1989). Journal of applied crystallography, 22(5), 483-497.

Hofmann, F., Abbey, B., Connor, L., Baimpas, N., Song, X., Keegan, S. \& Korsunsky, A.-M. (2012a). International Journal of Materials Research, 103(2), 192-199.

Hofmann, F., Song, X., Abbey, B., Jun, T.-S. \& Korsunsky, A. M. (2012b). Journal of synchrotron radiation, 19(3), 307-318.

Ice, G. E. \& Larson, B. C. (2000). Advanced Engineering Materials, 2(10), 643-646.

Karthikeyan, T., Saroja, S. \& Vijayalakshmi, M. (2012). Materials Science Forum, 702, 544-547.

King, A., Reischig, P., Adrien, J., Peetermans, S. \& Ludwig, W. (2014). Materials Characterization, 97, 1-10.

Kurdzesau, F. (2019). Journal of Applied Crystallography, 52(1).

Lehmann, T., Trempa, M., Meissner, E., Zschorsch, M., Reimann, C. \& Friedrich, J. (2014). Acta materialia, 69, 1-8.

Leitenberger, W., Hartmann, R., Pietsch, U., Andritschke, R., Starke, I. \& Strüder, L. (2008). Journal of Synchrotron Radiation, 15(5), 449-457.

Lynch, P., Barnett, M., Stevenson, A. \& Hutchinson, B. (2017). Metallurgical and Materials Transactions A, 48(11), 5206-5210.

Lynch, P., Stevenson, A., Liang, D., Parry, D., Wilkins, S. \& Tamura, N. (2007). Review of Scientific Instruments, 78(2), 023904.

Lynch, P., Stevenson, W., Fox, D., Ghaderi, A., Xie, L. \& Barnett, M. (2019). Available at SSRN 3446901.

MacDowell, A., Celestre, R., Tamura, N., Spolenak, R., Valek, B., Brown, W., Bravman, J., Padmore, H., Batterman, B. \& Patel, J. (2001). Nuclear Instruments and Methods in Physics Research Section A, 467, 936-943.

Marin, C., Cintas, A. \& Dieguez, E. (1994). Journal of Applied Crystallography, 27(5), 846-852.

Micha, J.-S. et al. (2012). URL http://sourceforge.net/projects/lauetools.

Nye, J. (1953). Acta Metallurgica, 1(2), 153-162.

Obstalecki, M., Wong, S. L., Dawson, P. R. \& Miller, M. P. (2014). Acta Materialia, 75, 259-272.

Pagan, D. C. \& Miller, M. P. (2014). Journal of Applied Crystallography, 47(3), 887-898.

Poulsen, H. F. (2004). Three-dimensional X-ray diffraction microscopy: mapping polycrystals and their dynamics, vol. 205. Springer Science \& Business Media.

Preuss, E., Butz, R. \& Krahl-Urban, B. (1974). Laue Atlas. Bertelsmann Univ. Verlag.

Proudhon, H., Guninchault, N., Forest, S. \& Ludwig, W. (2018). Materials, 11(10).

URL: http://www.mdpi.com/1996-1944/11/10/2018

Reed, R. C. (2008). The superalloys: fundamentals and applications. Cambridge university press.

Ren, Z., Bourgeois, D., Helliwell, J. R., Moffat, K., Srajer, V. \& Stoddard, B. L. (1999). Journal of Synchrotron Radiation, 6(4), 891917.

Riquet, J.-P. \& Bonnet, R. (1979). Journal of Applied Crystallography, 12(1), 39-41.

Russell, K. G. \& Ashby, M. (1970). Acta Metallurgica, 18(8), 891901.

Scheel, H. J. (2000). Journal of Crystal Growth, 211(1-4), 1-12.

Shade, P. A., Musinski, W. D., Obstalecki, M., Pagan, D. C., Beaudoin, A. J., Bernier, J. V. \& Turner, T. J. (2019). Current Opinion in Solid State and Materials Science, 23(5), 100763.

Shokr, M., Tosson, A., Abboud, A., Algashi, A., Schlosser, D., Hartmann, R., Klaus, M., Genzel, C., Strüder, L. \& Pietsch, U. (2019). Journal of Synchrotron Radiation, 26(5).

Simons, H., King, A., Ludwig, W., Detlefs, C., Pantleon, W., Schmidt, S., Snigireva, I., Snigirev, A. \& Poulsen, H. (2015). Nature Communications, 6. 
Skarzynski, T. (2013). Acta Crystallographica section D, 69(7), 12831288.

Tamura, N. (2014). In Strain and dislocation gradients from diffraction: Spatially-Resolved Local Structure and Defects, pp. 125155. World Scientific.

Van Swygenhoven, H., Schmitt, B., Derlet, P., Van Petegem, S., Cervellino, A., Budrovic, Z., Brandstetter, S., Bollhalder, A. \& Schild, M. (2006). Review of Scientific Instruments, 77(1), 013902.

Van Swygenhoven, H. \& Van Petegem, S. (2013). Materials Characterization, 78, 47-59.

Warren, B. E. (1937). Journal of Applied Physics, 8(10), 645-654.

Warren, B. E. (1969). X-ray Diffraction. Courier Corporation.

Wenk, H. R., Heidelbach, F., Chateigner, D. \& Zontone, F. (1997). Journal of Synchrotron Radiation, 4(2), 95-101.

Wong, S. L., Park, J.-S., Miller, M. P. \& Dawson, P. R. (2013). Computational Materials Science, 77, 456-466.

Wright, S. I. \& Adams, B. L. (1992). Metallurgical Transactions A, 23(3), 759-767.

Wyckoff, R. W. G. \& Merwin, H. E. (1924). American Journal of Science, 8(48), 447-461.

iucr

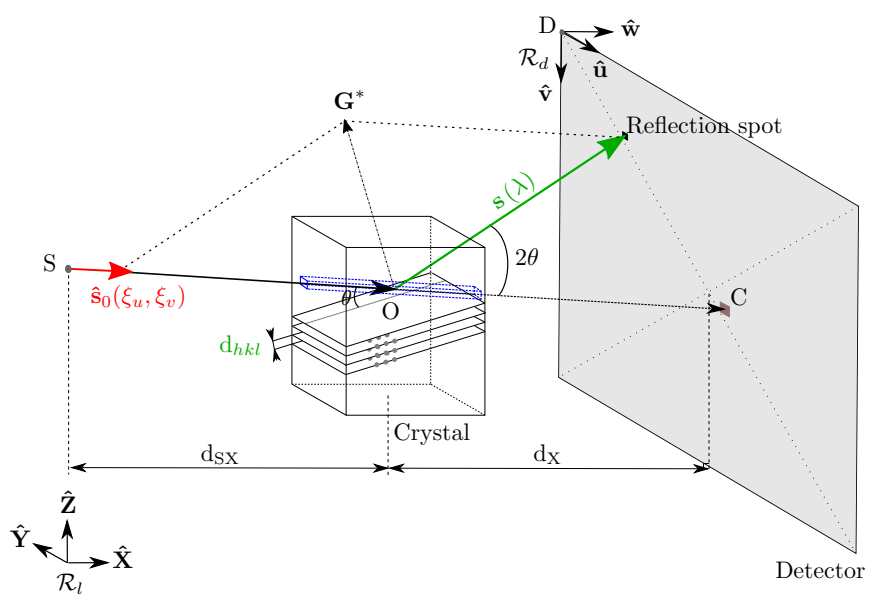

\section{Figure 1}

Schema of Laue transmission geometry for a given incident beam $\hat{\mathbf{s}}_{0}$ direction. The illuminated volume has been framed in blue.

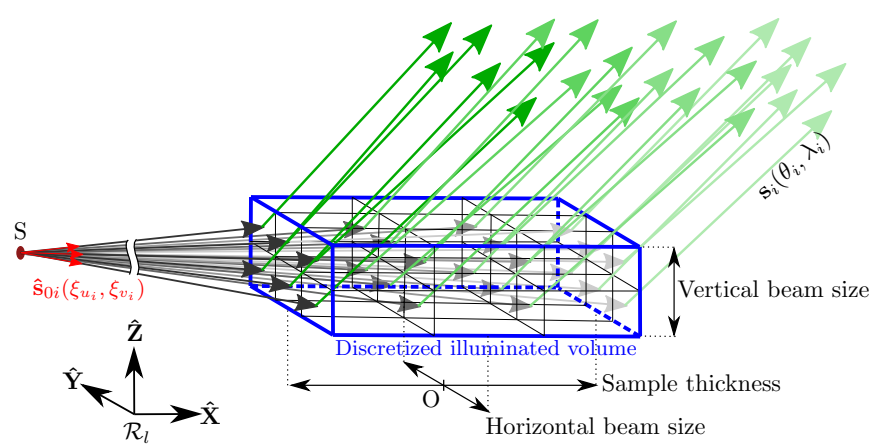

Figure 2

Schema of Laue transmission geometry used on a discretized illuminated sample volume. Diffraction has been applied on each voxels $i$ composing the volume. The difference between individual diffracting arrows has been exaggerated.

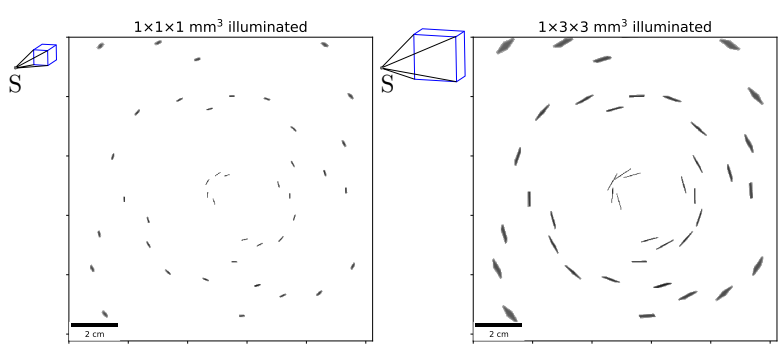

(a)

(b)

Figure 3

Forward simulation of an extended sample of nickel single crystal in Laue focusing condition with $\mathrm{d}_{\mathrm{SX}}=\mathrm{d}_{\mathrm{X}}=100 \mathrm{~mm}$ with $\hat{\mathbf{s}}_{0}\left(\xi_{u}=-3^{\circ}, \xi_{v}=2^{\circ}\right)$, voxel size $=10^{-4} \mathrm{~mm}^{3}$.

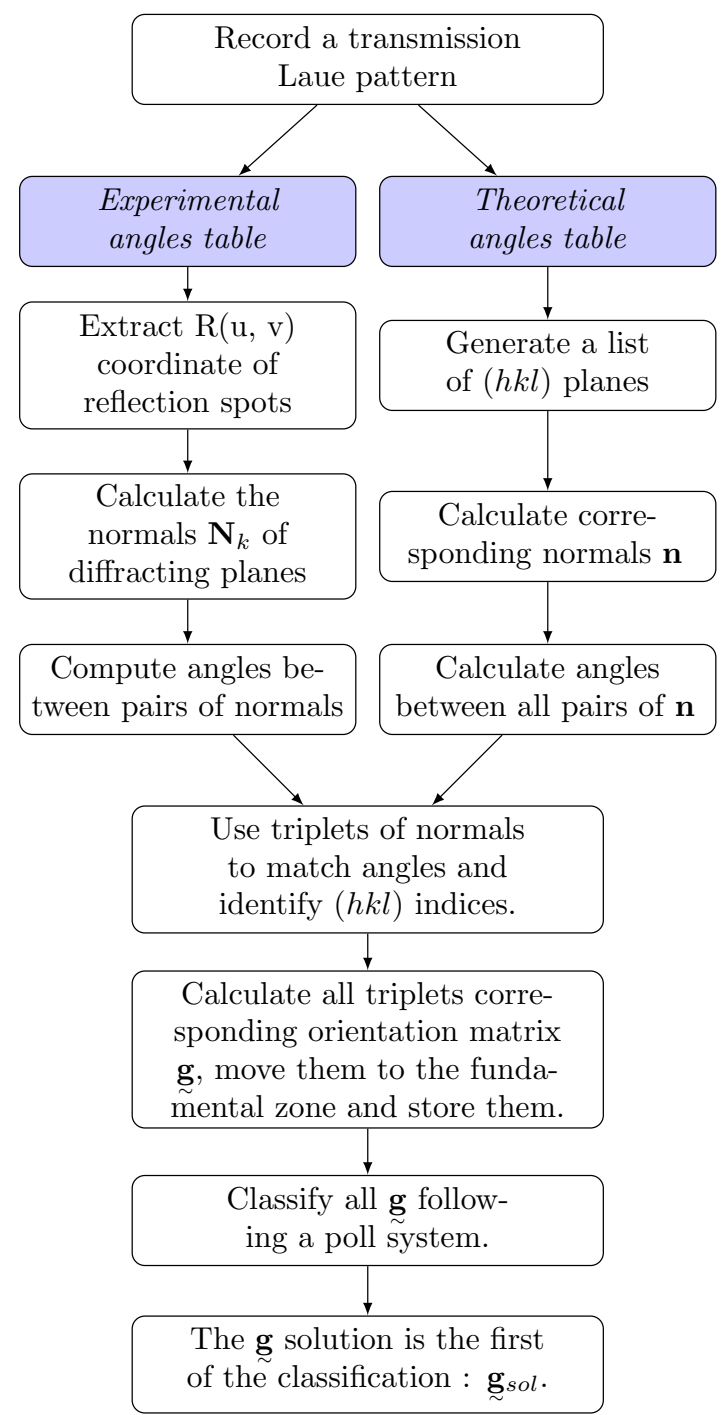

Figure 4

Flow chart of the Laue transmission pattern indexing algorithm. 


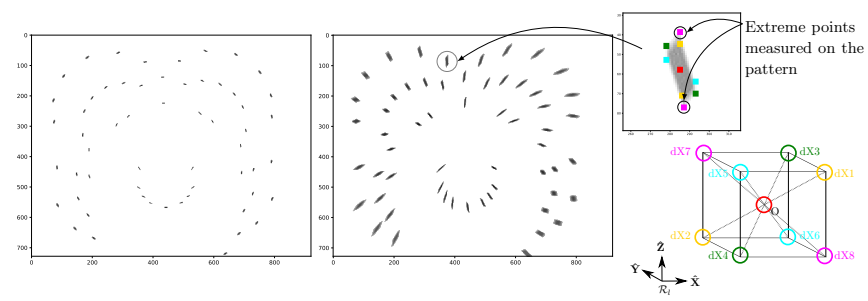

Figure 5

Simulated diffraction patterns of a pure nickel crystal oriented $\left(100.5^{\circ}, 10^{\circ}\right.$, $36.5^{\circ}$ ), in Laue focusing condition. Left simulation represents a perfect virtual sample while on the right a curved crystal lattice is used (see Eq. (15)). The lattice curvature causes spot spreading (asterism). Diffracting point position of all vertices of the illuminated volume is displayed for an example of spot. For each spot, only one diagonal of the cube is corresponding to the maximal spot spreading, and so the maximal curvature.

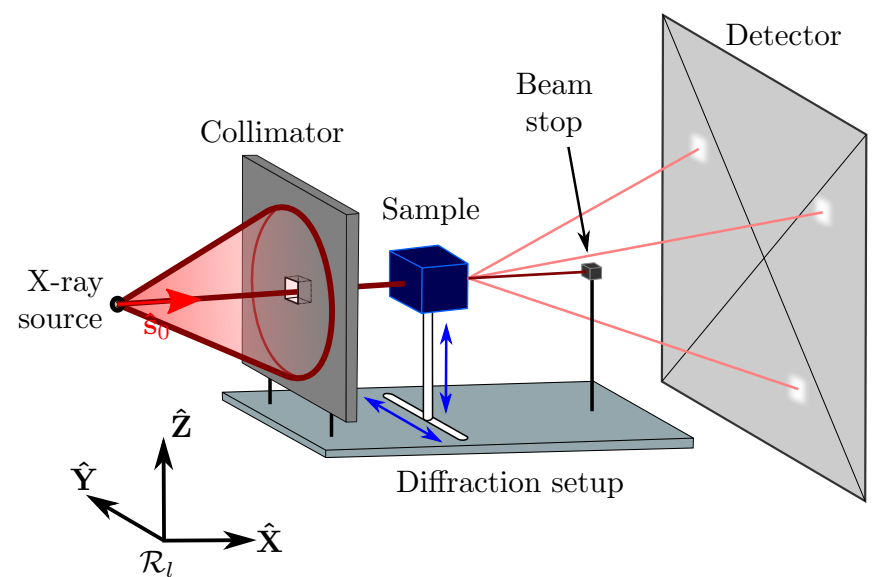

Figure 6

Sketch of Laue diffraction transmission setup.

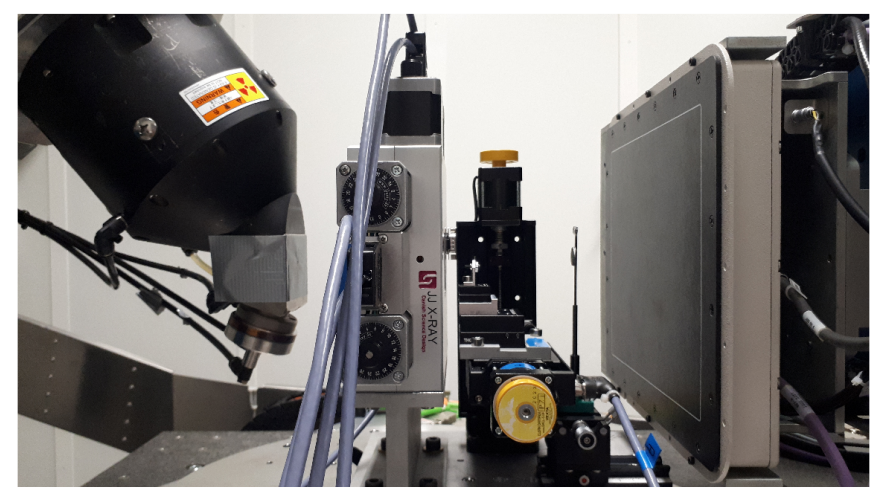

(a) X-ray system 1

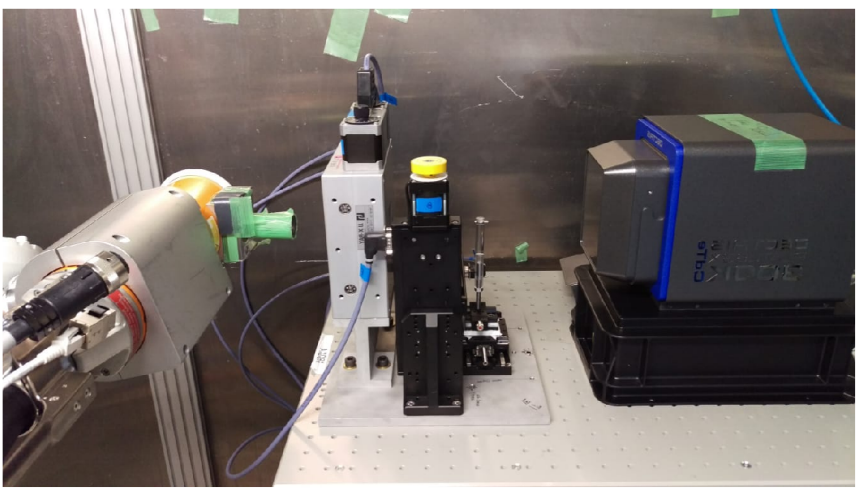

(b) X-ray system 2

\section{Figure 7}

Laue transmission diffraction setup portable: (a) at the tomographic platform of Navier Laboratory; (b) at DECTRIS X-ray cabin. From left to right : X-ray tube, slits collimator, sample positioning system, beam stop, X-ray detector.

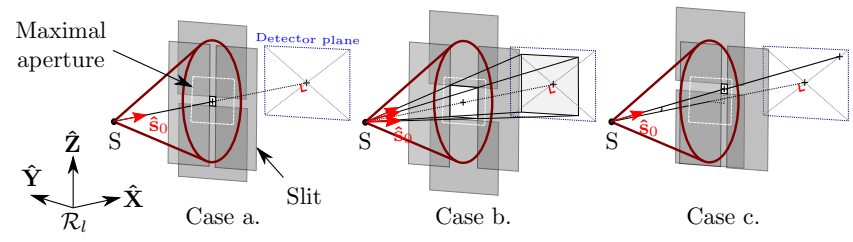

Figure 8

Examples of slits positions. Slits allows to point the incident beam normal to the detector plane, case a. Slits control the divergence of the beam and the illuminated sample volume, case b. shows a large aperture. Case c. depicts slits position directing the incident beam in a given direction. 


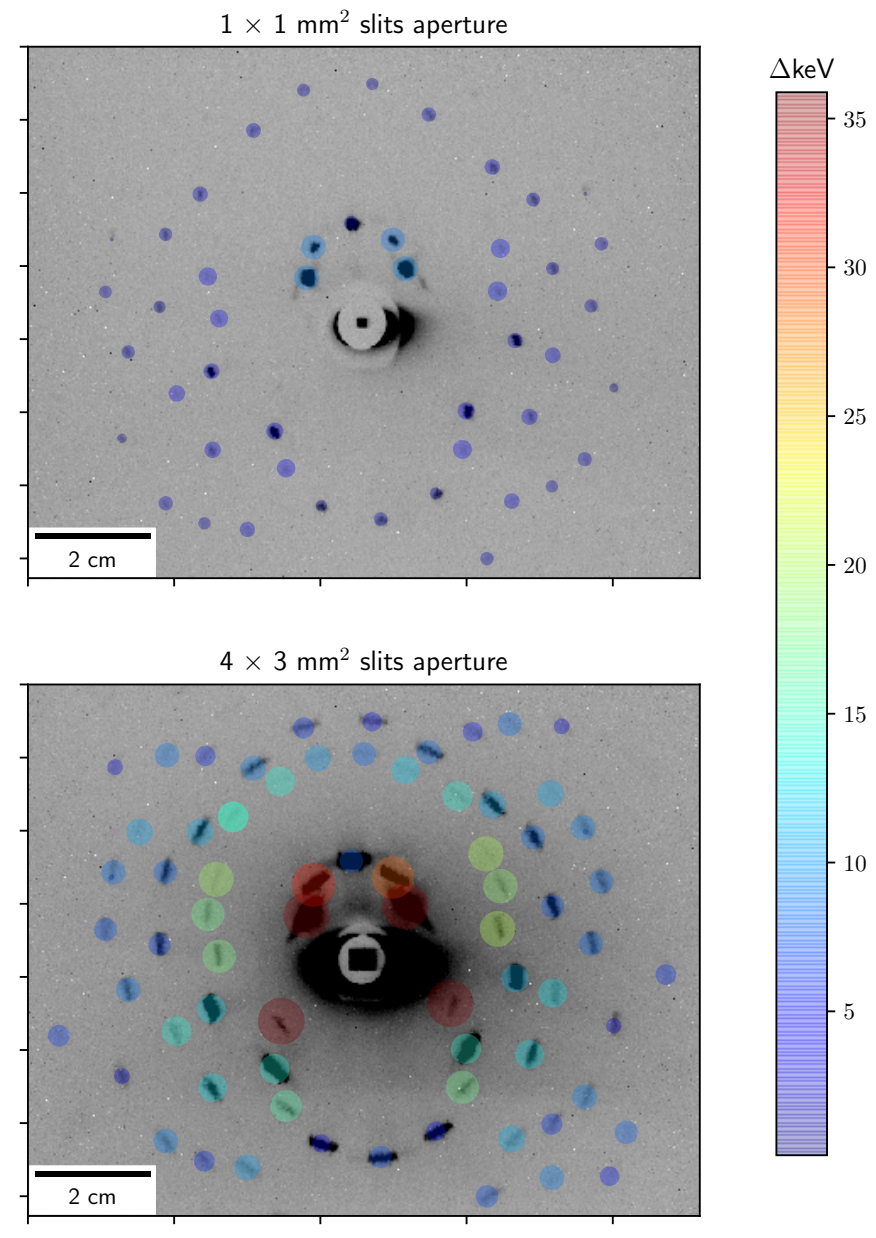

\section{Figure 9}

Laue transmission patterns of nickel-based alloy, oriented at $\left(91.1^{\circ}, 84.9^{\circ}\right.$, $84.3^{\circ}$ ), with a calibrated geometry of $\mathrm{d}_{\mathrm{SX}}=130 \mathrm{~mm}, \mathrm{~d}_{\mathrm{X}}=152.55 \mathrm{~mm}$, recorded for 2 slits aperture of $1 \mathrm{~mm}^{2}$ and $12 \mathrm{~mm}^{2}$. Superimposed scatter plots represent the range of energy involved on each reflection which is shown to increase with the beam divergence.

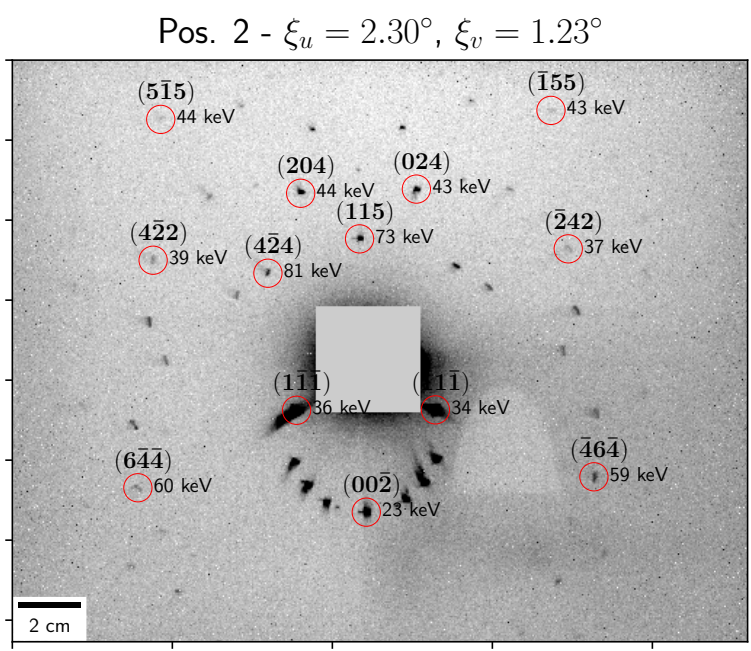

Pos. $4-\xi_{u}=0.44^{\circ}, \xi_{v}=1.21^{\circ}$
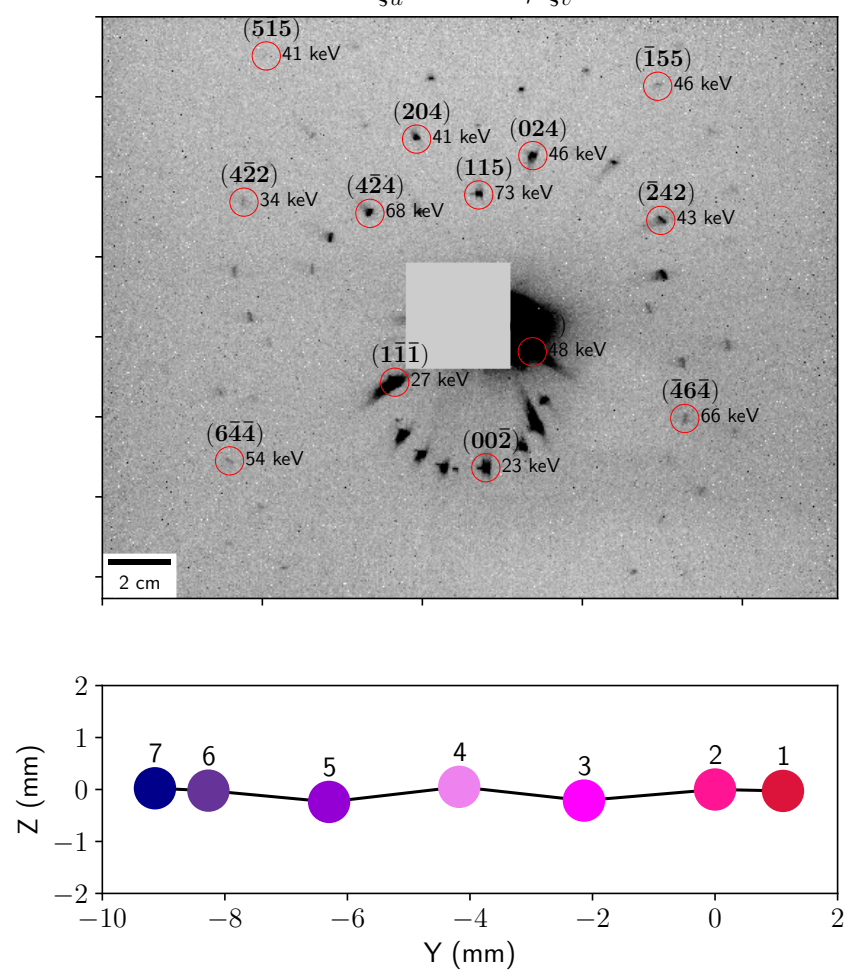

Figure 10

Laue transmission patterns of nickel recorded for a crystal orientation of $\left(100.49^{\circ}, 9.96^{\circ}, 36.5^{\circ}\right)$, with 7 different $\hat{\mathbf{s}}_{0}$ directions. The diffracting energies are modified with the change in direction of the incident beam. The indexing of the patterns also allows to get the corresponding 7 sample positions, here plotted on (YZ) plane. 


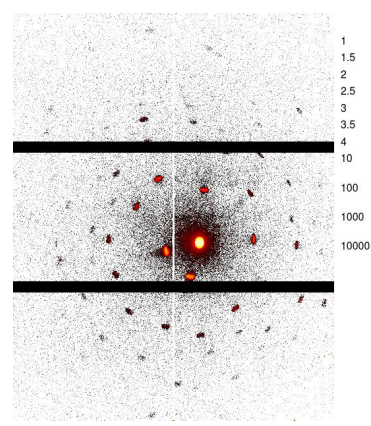

(a)

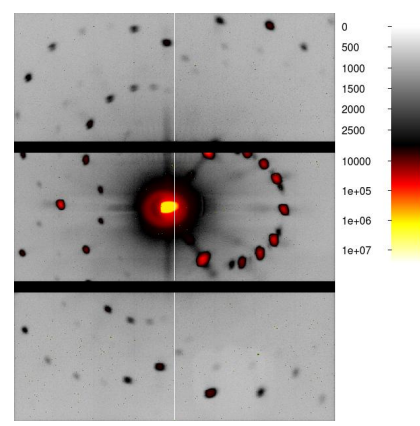

(b)

\section{Figure 11}

Laue transmission patterns of $1 \mathrm{~mm}$ nickel-based performed with the direct detector DECTRIS PILATUS3 X CdTe without beam stop. Acquisition parameters are: (a) $120 \mathrm{kV}, 5 \mathrm{~mA}, 0.1 \mathrm{~s}$ of exposure time, $\mathrm{d}_{\mathrm{X}}=70 \mathrm{~mm}$; (b) $160 \mathrm{kV}$, $25 \mathrm{~mA}, 1 \mathrm{~s}$ of exposure time, $\mathrm{d}_{\mathrm{X}}=102.2 \mathrm{~mm}$; both geometry are not in focusing conditions.

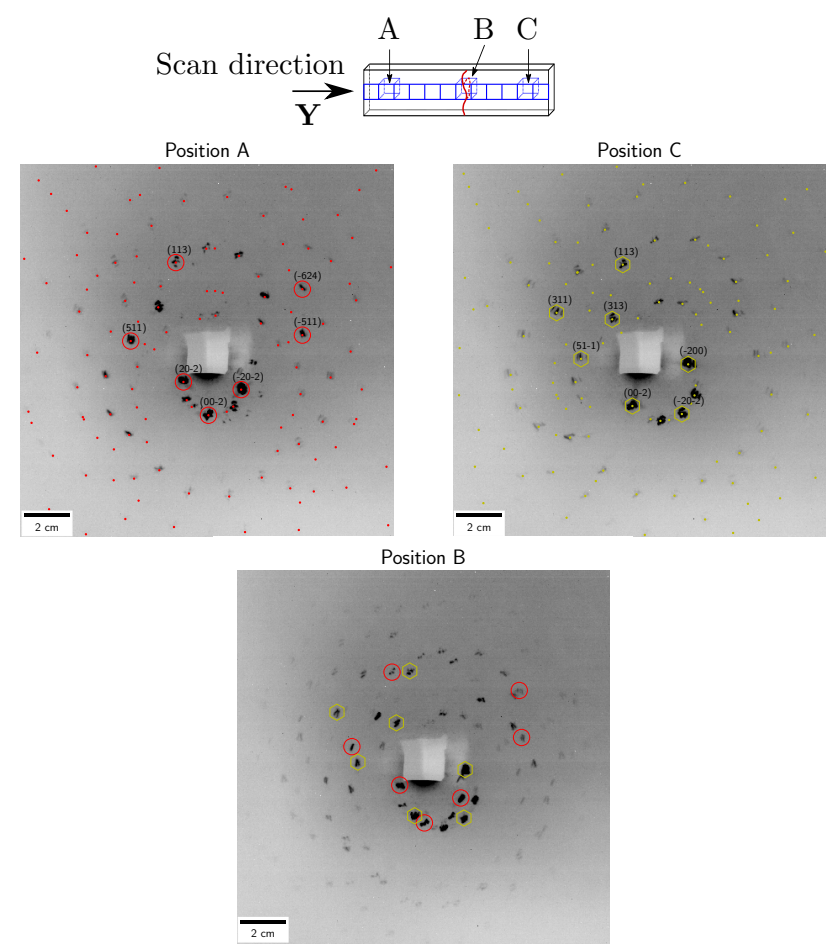

Figure 13

Detection of a grain boundary in a nickel-based alloy $1 \mathrm{~mm}$ thick sample. Misorientation between the two grains is $10.1^{\circ}$.
Figure 12

Laue transmission patterns of $1 \mathrm{~mm}$ nickel-based, $1 \mathrm{~s}$ of exposure time, $\mathrm{d}_{\mathrm{X}}=$ $102.2 \mathrm{~mm}$, not in focusing condition. Detection of local crystalline misorientation within a volume comprises in the illuminated one $16 \mathrm{~mm}^{3}$ of nickel-based. Acquisition parameters
Position B

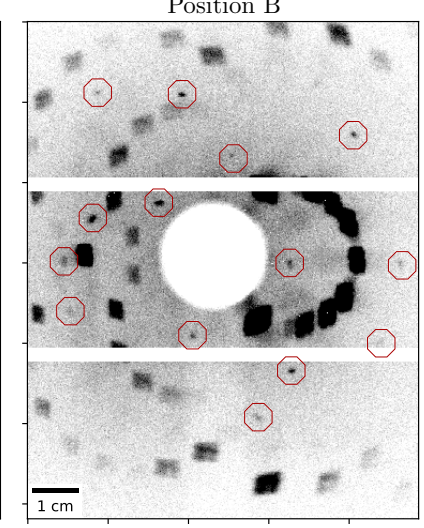



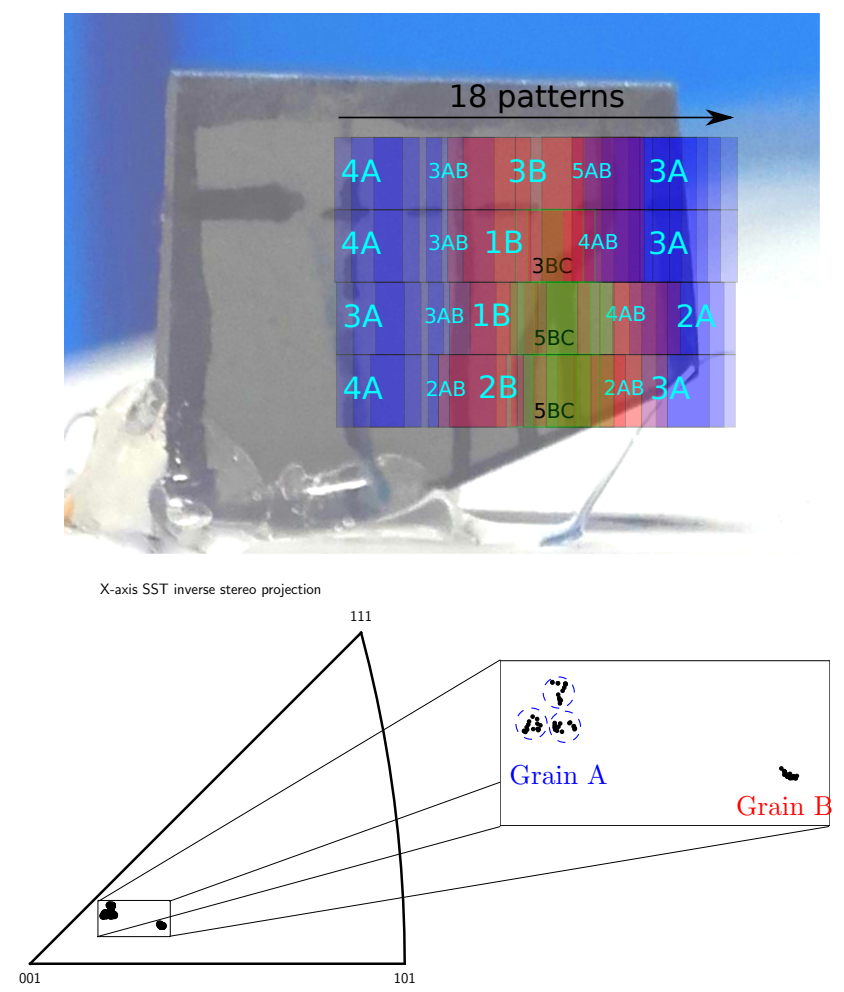

\section{Figure 14}

Orientation mapping of a tricrystalline nickel-based sample using a rectangular grid of 72 patterns. Orientations results are plotted in the inverse pole figure where grain A orientations can be clustered in 3 sub-grains.
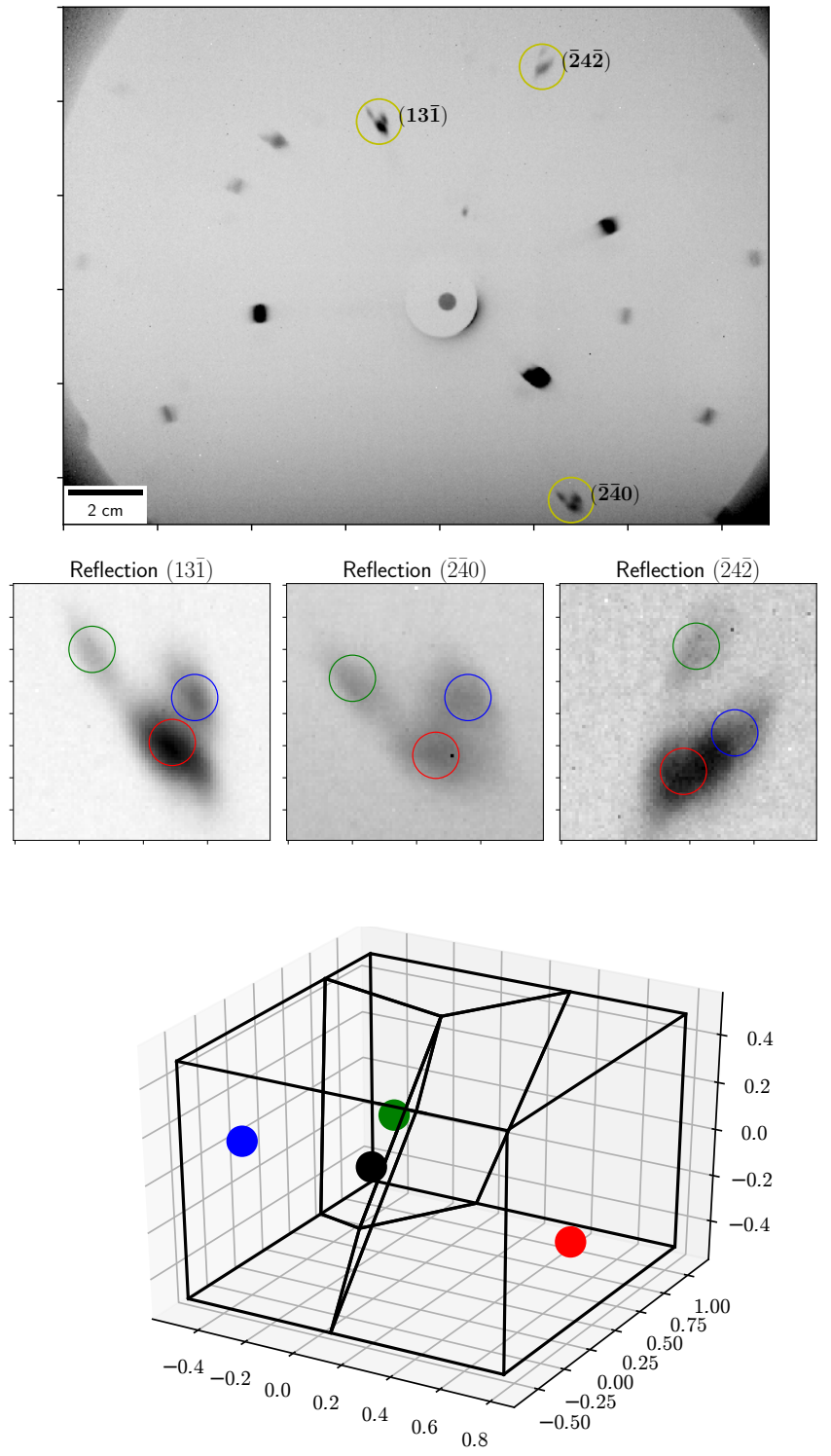

Figure 15

Laue transmission pattern of nickel-based alloy, oriented $\left(96.3^{\circ}, 103.4^{\circ},-3.8^{\circ}\right), \mathrm{d}_{\mathrm{X}}=205.7 \mathrm{~mm}$, not in focusing condition. Results of the sub-spot clustering to recover the positions of the sub-grains within the illuminated volume of $\sim 2 \mathrm{~mm}^{3}$, a Laguerre tessellation is used to get the sub-grain proportions. 


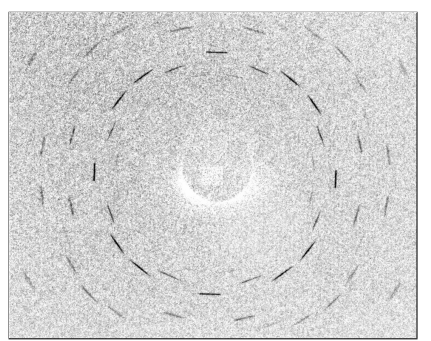

(a) $0.5 \mathrm{~mm}$ Silicon.

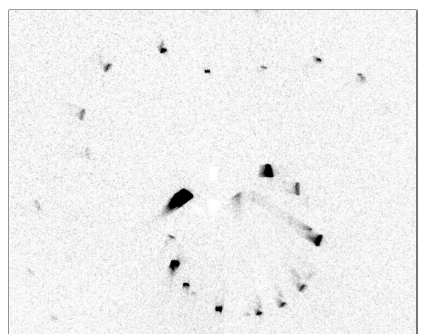

(c) $1 \mathrm{~mm}$ Nickel.

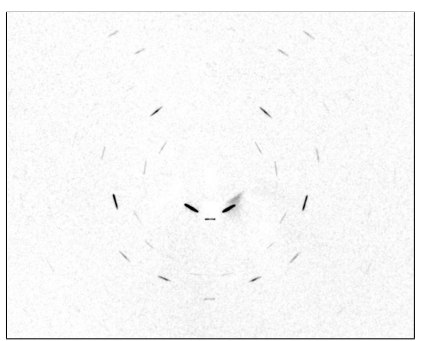

(b) $1 \mathrm{~mm}$ Germanium.

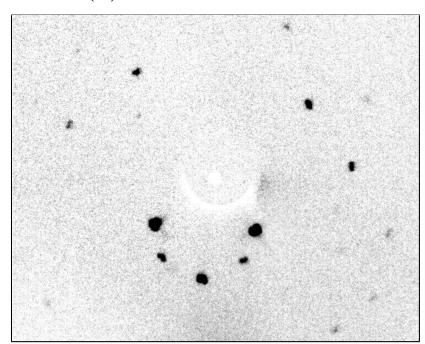

(d) $1 \mathrm{~mm}$ Nickel based alloy.

\section{Figure 16}

Laue diffraction patterns of single crystal samples with fixed acquisition parameters: $\mathrm{d}_{\mathrm{SX}}=\mathrm{d}_{\mathrm{X}}=125 \mathrm{~mm}$ (focusing condition) and X-ray source settled at $120 \mathrm{kV}$. For a fixed acquisition system, spots shape differences are observed: rod shape for silicon and germanium samples, rounded shape for nickel and nickel-based sample. 